\title{
Facilitating Space Operations via Documentation Management
}

\author{
Dr. Nadine Perera ${ }^{1}$ \\ German Aerospace Center, Wessling-Oberpfaffenhofen, Bavaria, 82234
}

\begin{abstract}
A substantial part of space operations consists in creating and editing documentation, be that design documents, flight and ground procedures, interface control documents, requirements documentation or technical notes and test procedures. An efficient and effective way to collaborate on and handle documentation linked to data in issue tracking, requirements management and configuration management tools is presented here to guarantee smooth space operations while considering the challenges that arise from security requirements.
\end{abstract}

\section{Nomenclature}

$C I \quad=$ Configuration Item

$C I D L=$ Configuration Item Data List

$C M=$ Configuration Management

$C M S=$ Configuration Management System

ColCC $=$ Columbus Control Center

$D M=$ Documentation Management

$D M S=$ Documentation Management System

GITS = GSOC Issue Tracking System

$G S O C=$ German Space Operations Center

$I P \quad=$ Internet Protocol

$I T=$ Information Technology

$L D A P=$ Lightweight Directory Access Protocol

$M U S C=$ Microgravity User Support Center

$O C R=$ Optical Character Recognition

$U S O C=$ User Support Center

$V S-N f D=$ „Verschlusssache - Nur für den Dienstgebrauch“/ Classified - For official use only

\section{Introduction}

$\mathrm{Q}$ UALITY Management and Configuration Management (CM) are especially important in space operations as compared to other fields. This is due to the highly regulated nature of space operations, which in turn results from the fact that a substantial part of the system in question cannot be accessed after the launch, being in space. Documentation Management (DM) is an important part of CM. For any space mission, a substantial part of the workload of building a space operations center consists of documenting that process and efficiently managing the resulting documentation, from design documents, flight and ground procedures, interface control documents, requirements documentation to technical notes and test procedures. All these documents will be edited by a variety of personnel collaborating on them. Typically, several persons are required to review, approve, and sign the documents. This often leads to many emails with attached documents being sent to and fro, thus creating local copies of the documents in question which increases the confusion as to which document is the latest, current, applicable one.

1 Configuration Manager, Quality, Configuration and Security Management, Münchner Straße 20, WesslingOberpfaffenhofen, Bavaria, 82234, Nadine.Perera@dlr.de

1

American Institute of Aeronautics and Astronautics 
An efficient documentation management process, supported by a user-friendly Documentation Management System (DMS) can greatly enhance the quality of the work undertaken. Documentation typically needs to be linked to information in other systems, such as issue tracking systems, requirements management systems and configuration management systems. A consistent way of managing this distributed information in diverse systems is crucial to guarantee efficient space operations. This paper presents the approach taken at GSOC to successfully implement and introduce a user-friendly DMS while managing space operations related constraints.

\section{Expected Benefits, Constraints, and Resulting Objective}

The introduction of a DMS, just like that of any IT system, is motivated by a number of expected benefits. However, handling documentation within a central IT tool will not come without a cost, and certain constraints must be managed. Some of the constraints result from the nature of a space operations center, e.g., the restricted access to internal networks from external locations. Some constraints are independent of the field in which a DMS is introduced, e.g., the fact that a general process will always need to be the common denominator of the currently practiced processes. This results in the fact that some users will be discontented, since they are forced to abandon their pet process or tool. In order to decide if the introduction of a DMS was successful, we shall analyze the expected and verified benefits of a DMS, both before and after the introduction of the system. Hence, the state and process of documentation before and after the introduction of the system shall be defined.

\section{A. Documentation Handling Status Before the DMS Introduction}

The status of project documentation management before the introduction of the DMS was as follows: Most project documents are placed on file shares. For some missions, the document numbers, version numbers and release dates, sometimes also the initials of the last person to change that document, are concatenated to the document file name in order to be able to keep track of changes and document versions. This results in many document files, which are really different versions of one document. Document identifiers are typically generated in an MS Excel sheet by the documentation manager of the project. Most space missions have their own conventions regarding the form of the document version (e.g., version numbers of the shape 1.A or 1.1). For the signature and review workflow, the document is typically attached to one or several emails and sent to the involved users. Some missions use digital signatures, some missions print out the cover page of a document, have the required persons sign it, then scan the front page and replace the first page on the PDF version of the document with the signed page. This may also involve printing the cover page with some signatures already on it, then signing it and scanning it again. Some missions use a makeshift workflow process by moving documents from folder to folder on a file share as the document status changes. Outdated versions of documents are typically moved to a subfolder called "old" or "outdated." Documents can be deleted by any user who has access to the file share, which occasionally leads to disappearing documents and consequently double work.

Some missions use a Configuration Management System (CMS) for document management. Since documents can be configuration items (CIs), the CMS allows for documents to be created as CIs, with metadata such as title, description, version, author, release date, and with the document file attached. The CMS can create Configuration Item Data Lists (CIDLs) for all or subsets of CIs, such that a certain amount of DM is made possible. However, the document files in the CMS are only the released versions of the document (in MS Word and PDF format). The collaboration process during changes to the MS Word file, or checking documents out for editing and back in, is not supported at all by the CMS.

\section{B. Expected Benefits of a DMS}

Even before the beginning of the DMS introduction project, the general opinion was that a DMS was needed for space project documentation management due to the following benefits:

1. Having one central repository shall solve the problem of finding the latest version of a document, which leads to a lot of time consuming comparisons of different document versions, and in some cases makes it difficult to find documents at all.

2. Version control and history, i.e., grouping documents such that the previous versions of the document can be accessed from one document provides the benefit of structure and uncluttered navigation. The alternative is having copies of all the different document versions distributed over several storage locations. With version history, the user will always know which document version is the latest one, how old it is, by whom it was edited last, and how its history has progressed. 
3. Document numbering support by the tool will be greatly useful in order to ensure that only unique document numbers are generated, and that document identifiers will conform to the prescribed format.

4. Full-text search is theoretically possible on file shares via the Windows Explorer, however, it does not seem to be very reliable. Full-text search on a DMS is one of the main advantages, and, especially if combined with an OCR-engine to recognize the text from scanned PDF files, enables the user to efficiently find the document she is looking for.

5. Tracking/Traceability shall greatly improve with a DMS, such that it becomes clear who edited which document in which way, when and, if the user left a check-in-comment, even why. All changes, both to the document content and the document metadata, are tracked in the DMS.

6. Workflows including digital signatures shall be supported by the DMS, such that the tedious process of emailing documents to and fro, which also clutters user's mailboxes, will become obsolete.

7. Role-based access models will help the user to structure the information classification of the documents in a way that supports the mission goals and that can be generalized across projects. The ability of inheriting access rights from top-level folders or setting them individually allows for a very fine granularity of access rights combinations, which is transparent to the users. Also, by hiding folders from the user who has no access rights to it, information security will improve, since malicious users will not even see a folder to which they might want to gain illegal access.

8. Further, a DMS shall generally improve collaboration by showing the history of a document together with information on the editors of the document, and by enabling the user to check documents out for editing, and check them back in again as a new version.

9. Expected benefits of the DMS are also the automatic comparison of document versions and

10. the (concurrent) co-editing of documents, meaning that more than one user could edit a document at the same time, as it is possible with Google Docs documents.

\section{Expected Constraints on a DMS for Space Operations}

The expected constraints on the successful implementation of an integrated DMS were the following before the implementation:

1. Network-internal and-external access to the DMS is required, since the documents in the DMS shall be reachable both from within the GSOC Office LAN and from the Internet. The requirement to reach the DMS from the Internet arises both from the case of traveling members of GSOC, who shall be able to access their documents from external locations, and from the fact that external partners of GSOC and the external facility MUSC shall also be able to use the DMS. Due to an encapsulated and highly complex, concentrically structured, gated and secured operational network setting, which is disconnected from the Internet for security objectives, an approach must be found how the documents in the DMS can be accessed from outside the GSOC network without compromising the security requirements or heightening the probability of connection problems from the inside of the network.

2. Different user authentication protocols: Due to the heterogeneous mission structure at GSOC, divided between manned (ColCC) and unmanned missions (satellite and sounding rocket missions), many users have two login names with different passwords at the user authentication system, here an OpenLDAP. All users have an additional Microsoft ActiveDirectory login for authentication at DLR and email access. For the implementation of the DMS, the best-suited authentication and authorization system must be selected and possibly adapted.

3. Heterogeneous user groups in regard to levels of IT tool affinity require different amounts of training. With respect to the users' affiliation, the potential users are working for different employers, such as ESA, the ColCC USOCs (Columbus User Support Centers) all over the world, Airbus Defence \& Space, DLR, or subcontractors. The latter situation poses additional organizational challenges, since some users are placed on-site in the GSOC building, whereas some are working from external locations. Some users are internal personnel, whereas others are subcontracted, which must be reflected in the document access right levels.

4. Differently classified documents: The documents to be stored in the DMS have different information security classifications. Unclassified, open or internal documents may be accessed from outside the GSOC after login to the DMS, but documents with a higher information security classification, e.g., the German VS-NfD/ESA Restricted, must not be accessed from outside the GSOC and may be accessed only by users with the required security clearance. This includes users who administrate (parts of) the DMS or its storage locations.

5. Various office tools and operating systems are to be supported by the DMS, such as Linux and Windows, Firefox and Internet Explorer, Microsoft Office and Libre Office. If the DMS is a browser-based tool, it 
needs to be available for Firefox and Internet Explorer, such that it can also be used on Linux platforms. If the DMS is a client-based tool, clients for both Windows and Linux must be available.

6. Multiple current document storage locations, such that the documents will have to be migrated into the DMS from the CMS, from multiple file shares in different networks, and from users' personal hard disks. During the migration, the metadata must be kept in sync, and it must be determined which of the many different document versions is the latest and applicable one.

7. ECSS Standards ${ }^{2}$ require data to be related across different information systems, (e.g., CMS, issue tracking and change management tools), which requires to share data across network boundaries. For example, the CIDLs that are currently generated for document CIs from the CMS shall still be generated after some or all of those documents have been migrated to the DMS. Our issue tracking and change management tool, GITS (GSOC Issue Tracking System), shall allow the users to write discrepancy reports or change reports on documents in the DMS, such that an interface between the two systems needs to be defined and implemented.

8. 24h availability of the system is required, hence redundancy must be implemented on the network and hardware side for the documentation management servers and a switchover process must be defined.

9. In-house location for the documentation management servers shall be granted for availability and confidentiality objectives. Since the availability of the DMS will be crucial for operations, the DMS servers must not be located outside GSOC. This requirement may exclude some DMS providers.

10. A big reporting demand from the customer's side results in many deliverable documents and complex approval processes, which makes it important to provide effective workflows.

11. An already existing wide range of stand-alone solutions for document management used in different departments raises the challenge to integrate or otherwise address the existing functionalities.

12. Heterogeneous mission structures represent a constraint on the unifying effect of a generic DM process and DMS. Both manned missions compared to unmanned missions, and unmanned satellite missions compared to one another, vary greatly in their chosen document structure. Hence, it will be difficult to find a unifying document structure for the general project, however desirable that would be for the users who work with multiple projects.

The challenges listed above need to be addressed while implementing and introducing the DMS, and the matching DM process.

\section{Objective}

The objective of this paper is to present a successful implementation of an integrated Documentation Management System (DMS) at the German Space Operations Center (GSOC). In this context, "successful" is defined by the following three criteria:

1. A high percentage of contented users,

2. Consideration for the project management triangle defining the quality of the DMS as the balance of cost, time, and scope, and

3. Compliance with international Standards, e.g., the ECSS (European Cooperation for Space Standardization) Standard, which defines many aspects of the documentation management process for space projects.

We found that the third criterion is actually more of a constraint that we have accounted for than a criterion to measure the success of the DMS introduction project by. Therefore, the standards compliance is addressed in Sections III, and V., Design Constraints, whereas the first criterion is discussed in Section IV., and the project management triangle is discussed in Section V.

\section{Verified Benefits and Addressed Constraints}

\section{A. Documentation Handling Status After the DMS Introduction}

The status of project documentation management after the introduction of the DMS is as follows: Of the 8 satellite missions at GSOC, 6 have been migrated to project sites in the DMS. The only manned mission, Columbus, and the Mobile Rocket Base have their documents in one large site each. There are 12 more project sites in the DMS, representing general functions across missions, such as Mission Planning, Quality Management, or Configuration Management. 176 users have participated in 12 DMS trainings. 
At least all the formal project documents are placed in the projects' sites in the DMS. There is only one instance of each document, which contains all the previous versions of it. Thus, the file name of the document now consists purely of its title, with version number, document identifier, and last changing author being metadata information. The version number format is of the form x.y.z, with $\mathrm{x}, \mathrm{y}$, and $\mathrm{z}$ being natural numbers including zero. The last place of the version number, $\mathrm{z}$, is incremented by the DMS with every check-in of a document, $\mathrm{x} . \mathrm{y}$ denotes the version of the document as specified in the document itself. Version numbers of the format 1.A have been automatically changed to 1.1. The signature and review workflow allows the user to choose a number of users or user groups for the review process and a number of users for the signature and approval process, together with escalation dates. The progress of the workflow can be tracked in the DMS, tasks may be reassigned, and users who have a task receive an email reminder with the link to the document in the DMS and a link to the task that they have to complete. The signature process is completed with digital signatures.

Only site managers and the owners of a document have the right to delete documents. The group of users with access to the document can be seen in the DMS. In addition, a typical user rights hierarchy was created for most of the missions, with the project manager having access to all documents, the project personnel manager having access to personnel documents, internal project members having access to all but contractual and personnel documents, and external project members having access to the remaining documents. The documents have document identifiers which are consistent within the mission and specified with regular expressions. The document identifiers may be different for different missions, since the format is often dictated by the customer. The documents from the CMS have been migrated to the DMS in such a way that the metadata information is still available in the CMS and CIDLs may still be produced. The metadata is kept up to date automatically by pulling the metadata from the DMS in a continuous synchronization process. Collaboration on documents is supported by checking documents out and back in for editing and versioning them. An online edit function allows to edit MS Office documents with the Firefox browser without explicitly downloading them, and checks in the document on each save. While an MS Word document is checked out via online editing, the MS Word compare function can be used to compare the current version of the document to the other versions saved in the DMS. The result of the comparison is shown in MS Word's Track Changes Mode in a separate document. This allows users to quickly grasp the changes since the last version for documents they have to approve, such that they will only need to review the changed parts instead of reading the complete document.

\section{B. Verified Benefits of a DMS}

In the DMS Trainings, which have been started after the introduction of the DMS, we continuously ask the users beforehand about their opinion on what the benefits of a DMS are. They usually list the same benefits as the ones we had put together before, namely:

1. Having one central repository did markedly improve the finding of the latest version of a document, cf. Task 3 in Section IV.

2. Version control and history, as expected, has improved both objectively and subjectively, cf. Task 1 in Section IV.

3. Document numbering support by the tool has proven to be very useful, however, the process of entering the document identifier is still a bit cumbersome.

4. Full-text search is more reliable on the DMS than on the file share. However, due to problems with the DMS index and a rather complicated search syntax, the benefit could be greater. A future version of our DMS will change the search syntax to a "Google-like" one, and once the index problems have been solved, the full-text search will be even more helpful.

5. Tracking/Traceability has improved markedly with all the metadata shown by the DMS next to the document, and also due to the fact that the deletion of documents is limited to the DMS site managers and the document owners.

6. Workflows including digital signatures work as designed and are very useful, even though the complexity of the subject has lead to a number of problems, which had to be solved first.

7. Role-based access models have been employed to structure the information classification of the documents as expected, in a way transparent to the users.

8. The DMS has generally improved collaboration by showing document metadata, status, and version history, c.f. Tasks 22 and 23 in Section IV.

9. Whereas the automatic comparison of document versions could not be realized natively in the DMS, the MS Word comparison in conjunction with the online editing feature delivers exactly this benefit.

10. The concurrent co-editing of documents at the same time was supported by the chosen DMS for a while, with MS Office 2003, until we changed to the current MS Office 2010. We are still waiting to see if the 
feature will be implemented for MS Office 2010. None of the other available DMSs offered this feature at all.

\section{Addressed Constraints of the DMS}

The expected constraints on the successful implementation of an integrated DMS were the following before the implementation:

1. Network-internal and -external access is unfortunately still missing and the DMS can only be reached from the internal GSOC Office LAN, at present, not from the Internet. This accounts for some dissatisfaction of the users, especially those who travel much and those in external locations. We hope that the external access to the DMS can be implemented soon, after some amount of preliminary work, such as creating a risk analysis together with the Network Infrastructure Systems group, setting up a reverse proxy, a new network area which is accessible from both the inside and the outside of the network, and setting up an infrastructure for a two factor authentication system using one time passwords sent to cell phones.

2. Different user authentication protocols: For the DMS, one of the Open LDAPs was chosen and the users were configured accordingly. The problem of the two login names with different passwords is in the process of being solved, which will reduce the confusion of the users regarding which login/password combination to use to access the DMS.

3. Heterogeneous user groups were addressed with a wide variety of trainings, which were available for all users regardless of their employer. Once the external access is available, the user training will have to be made available to external users in a convenient way.

4. Differently classified documents: The DMS can manage documents with all kinds of information security classifications, as long as the document classification itself does not forbid storing the document in an IT tool, e.g., ESA Secret. Unclassified, open or internal documents may be accessed from outside the GSOC, once the external access is made possible. The documents with a higher information security classification, e.g., the German VS-NfD/ESA Restricted, must not be accessed from outside the GSOC. Therefore, the access to documents can be restricted using an IP address range. That range can be set such that the proxy server's IP address, which any user connecting from an external location will have, will be blocked from accessing certain classified documents. Also, access rights lists can be managed in the DMS, and documents with an information security classification of Confidential or higher may be accessed only by users with the required security clearance, as noted in the list. Users who administrate (parts of) the DMS or its storage location are prevented from accessing classified documents via an encrypted storage, such that the direct access to documents via the file system is blocked.

5. Various office tools and operating systems are supported by the DMS, such as Linux and Windows, Firefox and Internet Explorer, Microsoft Office and Libre Office. The chosen DMS is a browser-based tool and works with both Firefox and Internet Explorer. There is an additional client-based tool, Alfresco Drive for offline document management, which only exists for Windows. However, the required functionality is covered by the browser-based tool.

6. Multiple current document storage locations, and also the sheer amount of project documentation, resulted in a challenge for the DMS introduction. Everyone agreed that the current file shares should be tidied up before migrating the resulting, important, and latest documentation into the DMS. However, the more documentation a project had accumulated in the past, the longer it took to organize the documentation in its many locations, which often resulted in a much longer transition period for a mission or, in one case, to postpone the migration to the DMS indefinitely. For the migration from the CMS into the DMS, a program was developed, which even sets the available document metadata from the CMS automatically in the DMS and already creates the connection between the document in the DMS and the CI in the CMS, where the document's metadata shall be synchronized to.

7. ECSS Standards require data to be related across different information systems, (e.g., CMS, issue tracking and change management tools), which requires to share data across network boundaries. The metadata synchronization interface between the DMS and the CMS allows for the missions to continue generating their CIDLs for document CIs from the CMS after the documents have been migrated to the DMS. Our issue tracking and change management tool, GITS (GSOC Issue Tracking System), allows the users to write discrepancy reports or change reports on documents in the DMS in a newly introduced "GITS System Affected." This system within the issue tracking tool was used from the start, also to track deviations from the requirements before a system baseline was set. An external URL field in the DMS document metadata 
allows the user to create a link to a GITS report. An interface control document specifying the relations between the three systems was written and the interface implemented.

8. 24h availability of the system is required, hence redundancy was implemented on the network and hardware side for the documentation management servers by the Network Infrastructure Systems group. The redundant servers are placed in the prime and backup server locations of GSOC, the applications and the database servers run as virtual machines on an VMware ESXi hosting platform, and are set up to perform automatic switchovers if necessary. The redundant DMS file system is synchronized constantly.

9. In-house location for the documentation management servers was granted for availability and confidentiality reasons. Since the availability of the DMS is crucial for operations, the DMS servers must not be located outside GSOC. This excluded some DMS solutions which would have required external hosting.

10. The big reporting demand from the customer's side and its resulting many deliverable documents and complex approval processes were taken into account and an effective workflow was specified. Due to the approval workflow's complexity, this resulted in some problems with the workflow management when the DMS was introduced, and may explain some less than optimal grades in Task 19, Section IV., but altogether the implemented solution is a solid one.

11. An already existing wide range of stand-alone solutions for document management was analyzed during the DMS introduction project, and their features were included in the DMS requirements as far as possible. As none of the pre-existing DM solutions (none of which was a fully functional DMS, but rather improvised tools born out of necessity) satisfied the user requirements, a different solution was chosen and customized, namely the Alfresco DMS. Some useful pre-existing features, such as MS Word metadata fields in document templates specifying the issue und release numbers, document title and document identifier, were incorporated into the selected DMS solution. Therefore, the DMS is able to warn the user if she tries to set a document version number to a number that differs from the one specified in the document itself and in its metadata fields.

12. Heterogeneous mission structures turned out to be a rather big challenge, and the one that could not truly be solved by the DMS introduction, cf. Task 14 in Section IV. However, a small improvement was made in that regard, as evidenced by the user survey.

Therefore, we can state that the expected benefits have in general been verified and that nearly all the constraints have been addressed. This qualifies the DMS introduction project as successful. As announced in Section II.D., we also strove to measure the success of the introduction project with a survey of user satisfaction, cf. Section IV, and regarding the project management triangle, cf. Section V.

\section{Success Measured by High DMS User Satisfaction}

One approach to determine the success of the DMS introduction project is to analyze the number of projects and missions migrated to DMS, the number of DMS users, and the number of DMS users having had a DMS training. Of the 8 active satellite missions at GSOC, 6 have been migrated to project sites in the DMS - the remaining 2 missions have decided that they will not run long enough to reap the benefits of starting a migration now. The only manned mission, Columbus, and the mission Mobile Rocket Base have their documents in one large DMS site each. There are 12 more project sites in the DMS, representing general functions across missions, such as Mission Planning, Quality Management, or Configuration Management. 176 users have participated in 12 DMS trainings within the last year.

As announced in Section II., another means to measure the success of the DMS implementation and introduction project is to ask the users how satisfied they are with the DM process before and after the introduction. We shall strive to make this evaluation by comparing two surveys. 


\section{A. Two Surveys with DMS Users}

A survey was performed before the introduction of the DMS, asking how satisfied users are with the current processes for retrieving, preparing, editing, reviewing, approving, collaborating on, and distributing documents. One year after the roll-out of the DMS, the survey was repeated. The users were asked to compare the documentation management processes with and without the DMS regarding usability and grade them.

\begin{tabular}{|c|c|c|c|c|c|}
\hline$\#$ & Task & Survey 1 & Survey 2 & $\begin{array}{c}\text { Survey 2, } \\
\text { only DMS } \\
\text { Users }\end{array}$ & $\begin{array}{c}\text { Average } \\
\text { Grade } \\
\text { Improvement }\end{array}$ \\
\hline 1 & Version Control & 3.27 & 1.94 & 1.83 & 1.44 \\
\hline 2 & Applicable Documents & 3.57 & 2.66 & 2.77 & 0.80 \\
\hline 3 & Finding Latest Version & 3.49 & 2.12 & 2.08 & 1.41 \\
\hline 4 & Distributing Documents & 3.17 & 2.41 & 2.43 & 0.74 \\
\hline 5 & Working with latest version & 3.33 & 2.00 & 2.04 & 1.29 \\
\hline 6 & Comparing document versions & 3.76 & 2.70 & 2.23 & 1.53 \\
\hline 7 & Document Identifiers & 3.21 & 2.36 & 2.25 & 0.96 \\
\hline 8 & Document Status & 3.15 & 2.10 & 2.18 & 0.97 \\
\hline 9 & Applicability of Documents & 3.32 & 2.57 & 2.56 & 0.76 \\
\hline 10 & Knowledge of Structure & 2.89 & 2.39 & 2.42 & 0.47 \\
\hline 11 & General Structure & 3.32 & 2.82 & 2.80 & 0.52 \\
\hline 12 & Knowledge Requirements Area & 2.96 & 2.50 & 2.41 & 0.55 \\
\hline 13 & Structure in Area & 3.12 & 2.56 & 2.58 & 0.54 \\
\hline 14 & Generealized Structure across Missions & 3.64 & 3.19 & 3.38 & 0.27 \\
\hline 15 & Knowledge of Requirements Personal & 2.86 & 2.42 & 2.48 & 0.38 \\
\hline 16 & Knowledge of Requirements General & 3.25 & 2.78 & 2.86 & 0.39 \\
\hline 17 & Corporate Identity & 2.99 & 2.77 & 2.81 & 0.18 \\
\hline 18 & Corporate Identity in Documentation & 3.15 & 2.80 & 2.57 & 0.58 \\
\hline 19 & Releasing Documents & 3.32 & 2.22 & 2.14 & 1.18 \\
\hline 20 & Editing Documents & 2.93 & 2.12 & 2.13 & 0.80 \\
\hline 21 & Reviewing Documents & 3.03 & 2.17 & 2.13 & 0.90 \\
\hline 22 & Co-editing Documents & 4.05 & 2.89 & 2.79 & 1.26 \\
\hline 23 & Collaborating on Documents & 3.26 & 2.31 & 2.11 & 1.15 \\
\hline 24 & Creating Document Reports & 3.83 & 2.45 & 2.22 & 1.61 \\
\hline 25 & Average over Tasks & 3.29 & 2.47 & 2.42 & 0.86 \\
\hline
\end{tabular}

Table 1: Average Grades on Tasks: The average grades on the tasks from the 77 participants for Survey 1, next to the grades from the 36 participants of Survey 2. The other two columns indicate the grades from the 25 participants from Survey 2 who actually work with the DMS, and the difference in grades between their grades and the grades from Survey 1. 
Two surveys were conducted, Survey 1 (September 2013) before and Survey 2 (August 2015) after the introduction of the DMS. 77 users participated in Survey 1, whereas 36 users participated in Survey 2. The surveys were not conducted anonymously, hence we know that 23 users have participated in both surveys. The users were

\begin{tabular}{|r|l|r|r|r|}
\hline \multicolumn{1}{|c|}{ Task } & \multicolumn{1}{c|}{$\begin{array}{c}\text { Survey 1 } \\
\text { Both }\end{array}$} & $\begin{array}{c}\text { Survey 2 } \\
\text { Both }\end{array}$ & $\begin{array}{c}\text { Average } \\
\text { Grade } \\
\text { Improvement }\end{array}$ \\
\hline 1 & Version Control & 3.04 & 2.00 & 1.04 \\
\hline 2 & Applicable Documents & 3.36 & 2.78 & 0.58 \\
\hline 3 & Finding Latest Version & 3.58 & 2.10 & 1.49 \\
\hline 4 & Distributing Documents & 3.32 & 2.44 & 0.88 \\
\hline 5 & Working with latest version & 3.42 & 1.96 & 1.46 \\
\hline 6 & Comparing document versions & 3.50 & 3.00 & 0.50 \\
\hline 7 & Document Identifiers & 3.05 & 2.44 & 0.60 \\
\hline 8 & Document Status & 3.08 & 2.25 & 0.83 \\
\hline 9 & Applicability of Documents & 3.13 & 2.57 & 0.55 \\
\hline 10 & Knowledge of Structure & 2.71 & 2.48 & 0.23 \\
\hline 11 & General Structure & 3.29 & 2.86 & 0.43 \\
\hline 12 & Knowledge Requirements Area & 2.79 & 2.58 & 0.21 \\
\hline 13 & Structure in Area & 3.04 & 2.70 & 0.34 \\
\hline 14 & Generealized Structure across Missions & 3.73 & 3.55 & 0.18 \\
\hline 15 & Knowledge of Requirements Personal & 2.68 & 2.40 & 0.28 \\
\hline 16 & Knowledge of Requirements General & 3.21 & 2.95 & 0.26 \\
\hline 17 & Corporate Identity & 3.09 & 2.91 & 0.18 \\
\hline 18 & Corporate Identity in Documentation & 3.18 & 2.76 & 0.42 \\
\hline 19 & Releasing Documents & 3.41 & 2.21 & 1.20 \\
\hline 20 & Editing Documents & 2.96 & 2.24 & 0.72 \\
\hline 21 & Reviewing Documents & 3.09 & 2.32 & 0.78 \\
\hline 22 & Co-editing Documents & 4.04 & 3.15 & 0.89 \\
\hline 23 & Collaborating on Documents & 3.00 & 2.41 & 0.59 \\
\hline 24 & Creating Document Reports & 3.31 & 2.63 & 0.68 \\
\hline $\mathbf{2 5}$ & Average over Tasks & $\mathbf{3 . 2 1}$ & $\mathbf{2 . 5 7}$ & $\mathbf{0 . 6 4}$ \\
\hline
\end{tabular}

Table 2: Average Grades on Tasks for partificipants of both surveys, the document management tasks were rated with an average 3.21 in Survey 1 and with 2.57 in Survey 2, which still represents an improvement of 0.64 grades.

asked to rate document management tasks in German school grades from 1-6, 1 being very good, 6 being insufficient; "not applicable" was also possible.

On average, the participating users rated their satisfaction with the document management tasks with a 3.29 in Survey 1, whereas Survey 2 scored a 2.47, which represents an improvement of 0.82 grades, cf. Table 1 .

If we restrict Survey 2 to the subset of those users who said they were using the DMS today (25 of the 36 participants), the average document management tasks were rated with an 2.42, i.e., slightly better than including the users who still do not work with the DMS today, but were also allowed to participate in the survey.

Table 1 shows the average grades on the tasks from the 77 participants for Survey 1, next to the grades from the 36 participants of Survey 2. The other two columns indicate the grades from the 25 participants from Survey 2 who actually work with the DMS, and the difference in grades between their grades and the grades from Survey 1.

Regarding only the subset of users who participated in both surveys, the document management tasks were rated with an average 3.21 in Survey 1 and with 2.57 in Survey 2, which still represents an improvement of 0.64 grades, cf. Table 2.

Figure 1 shows the first three columns from Table 1 graphically - note that lower bars represent better grades! 
Analyzing the data further, we can see that the user satisfaction with the introduction of the DMS improved in different amounts depending on the document management tasks. These questions were included in both Surveys, the number indicates the task number in Tables 1 and 2 and in the Charts 1 and 2.

1. How do you grade the version control on documents in your area of work?

2. How far can you see from the current document structure which documents are applicable?

3. How do you grade the finding of documents, especially the finding of the latest version of a document?

4. How do you grade the distribution process, i.e., the announcement of a new document?

5. How well can you judge that you are working with the latest version of a document?

6. How do you grade the comparison between two document versions?

7. How do you grade the assignment and tracking of document identifiers?

8. How well can you judge the status of a document (e.g., Draft, Applicable Document, Rework, Obsolete)?

9. How well can you decide between applicable and not applicable documents?

10. How do you grade your own awareness about the document structure in your work area?

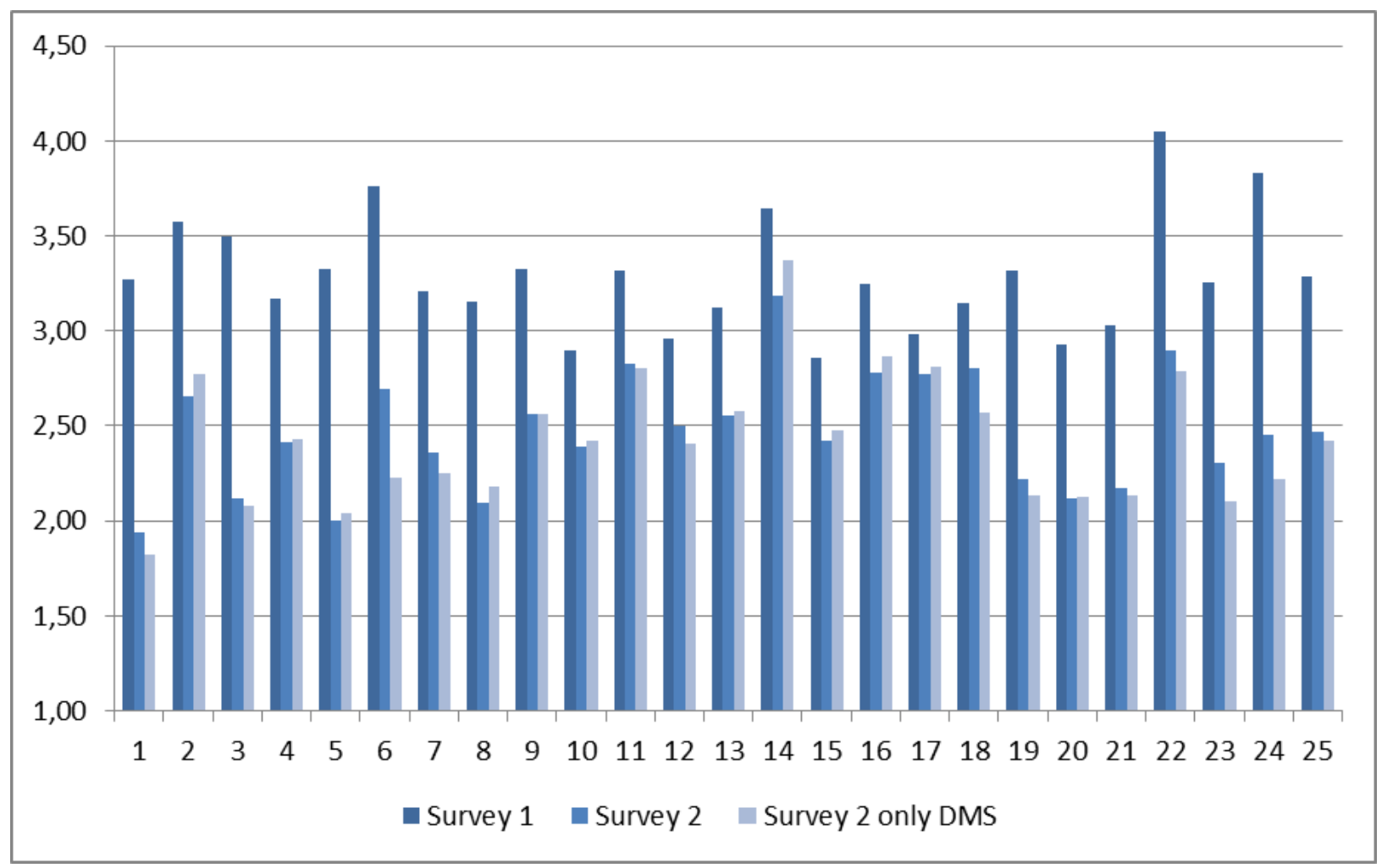

Figure 1: Average Grades on Tasks: note that shorter bars represent better grades! The last task represents the average over the 24 previous tasks.

11. How do you grade the general document structure?

12. How do you grade the access to knowledge and requirements for documentation in your area of work?

13. How do you grade the document structures in your area of work?

14. If you work in several projects, how far is the document structure similar, such that you know your way around the structure fast?

15. How do you grade your knowledge regarding requirements for documentation in you project?

16. How do you grade the access to knowledge regarding documentation requirements in general?

17. How do you grade your knowledge regarding Corporate Identity and the access to template documents?

18. How do you grade access to and compliance with Corporate Identity in the documentation process?

19. How do you grade the review and approval process, i.e., having others sign a documentk you authored?

20. How do you grade the review process, editing and improving of a document?

21. How do you grade the approval process, i.e., the signing of a document?

22. How do you grade the concurrent editing of documents together with colleagues?

23. How do you grade the collaborative editing of documents together with colleagues? 
24. How do you grade the creation of document reports?

25. Average over Tasks

The average improvement between the surveys, as listed in Table 1, is shown graphically in Figure 2. For our analysis why certain tasks have improved a lot, whereas others just improved a little, we will take a closer look at the improvements per task in the next Section.

\section{B. Discussion of Survey Results}

One interpretation for the fact that less than half as many users participated in the second survey is that the DM situation has improved substantially and therefore, less users are inclined to take the time to take a survey. The fact that the average grade of the participating users has improved with the DMS by $0.64-0.86$ grades also points in that direction, as well as the fact that no single task was rated worse than before the introduction of the DMS.

Task 24, the creation of document reports, has improved most by 1.61 grades, followed by Task 6 (comparing document versions) with 1.53 grades, Task 1 (version control) with 1.44 grades and Task 3 (Finding latest version) with 1.41 grades. The high improvement on these tasks comes as no surprise since all four tasks can be done with features which were specifically introduced with the DMS, and had to be done manually before the introduction.

Regarding the tasks where the improvement was smallest, we have Task 17 (Corporate Identity) with 0.18 grades, Task 14 (Generalized Structure across Missions) with 0.27 grades, followed by Task 15 (Knowledge of Requirements Personal) with 0.38, and Task 16 (Knowledge of Requirements General) with 0.39 grades. The fact that Task 17 yielded such a small improvement score may be due to the fact that the knowledge about Corporate Identity and where to find document templates was already high before the DMS introduction. Another explanation would be that not all users are aware of the template area in the DMS where the current templates are kept for easy

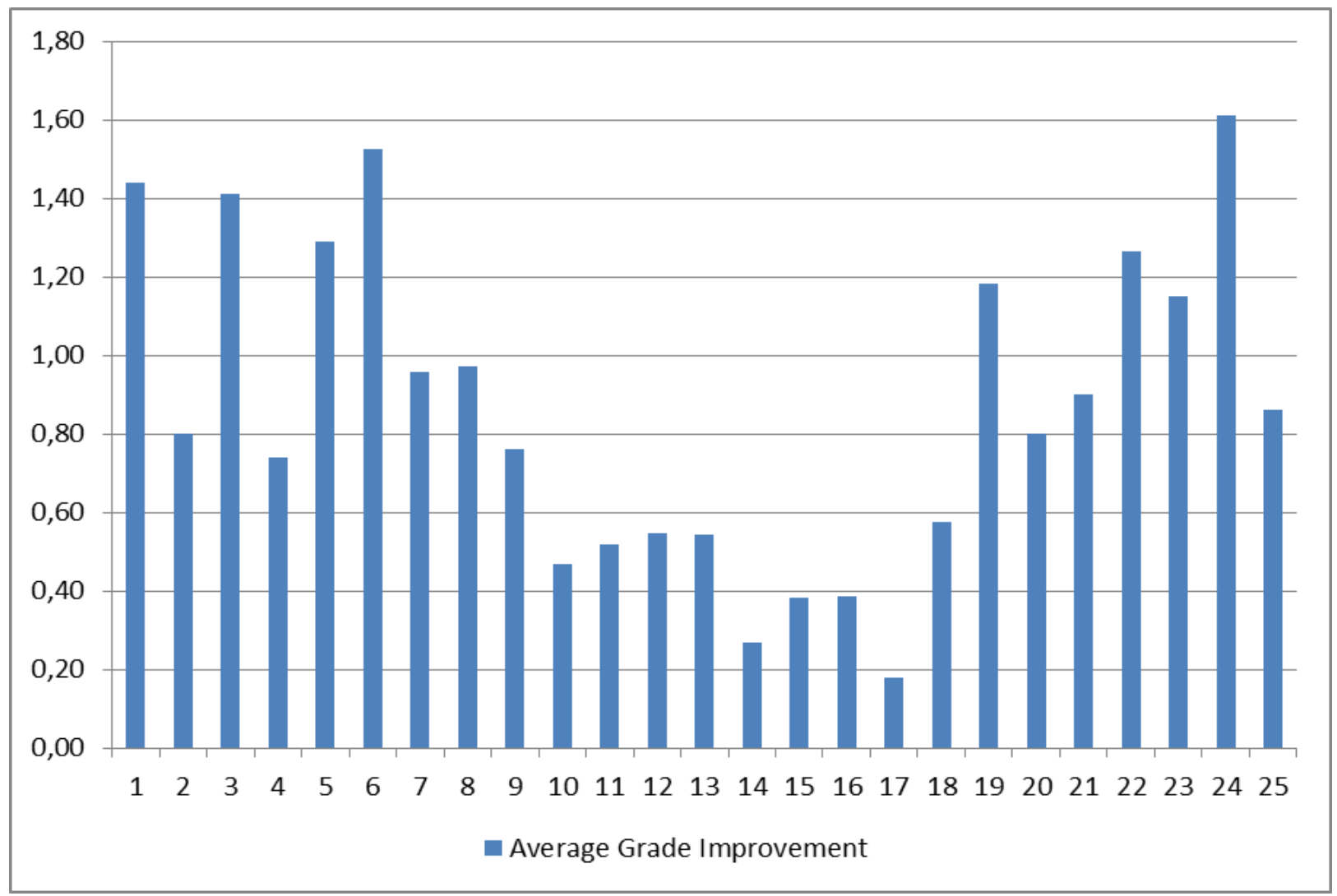

Figure 2: Average Grade Improvements over tasks.

access.

Task 14, the Generalized Structure across Missions, was expected to perform poorly, since we had several meetings and discussions in order to figure out a general structure, which just proved impossible at last. The common denominator between manned and unmanned missions, the amount and nesting depth of the different missions' documents in the DMS proved to be too small to be of use. Forcing all the missions to apply a generic 
structure to their documentation, regardless of the mission's number of subsystems and included documents, would have created too much overhead on the single mission. This would have outweighed the benefit for the users who have to participate in many different missions. Tasks 15 and 16 correlate with Task 14 in so far as the structure, especially a generic structure, would have helped the users to better understand the requirements for documentation, both in their project and in general. The situation being as it is, a mild positive trend is a good sign already.

In summary, the user surveys show that the DM process is better understood and perceived as more user friendly than before the introduction of the DMS. Hence the DMS introduction project may be judged successful in this aspect.

\section{Success Measured by Project Management Triangle}

The Project Management Triangle ${ }^{l}$ is a model of the constraints of project management. It places the three attributes scope, cost, and time on the corners of a triangle to show how they oppose each other mutually. The area inside the triangle represents the overall quality of the project. The triangle is used to illustrate that project management success is measured by the project team's ability to manage the project such that the expected results (scope) are produced while managing time and cost. It also reflects the fact that the three properties of a project are interrelated, and it is not possible to optimize all three one will always suffer.

In this example, the focus was set on the scope, since we thought it important to implement all the features required by the users in the requirements workshops (unless the requirements changed during the process, which also happened and was documented in requirements change management). The project cost was within the set limits, whereas the initially projected time did not suffice to finish the project.

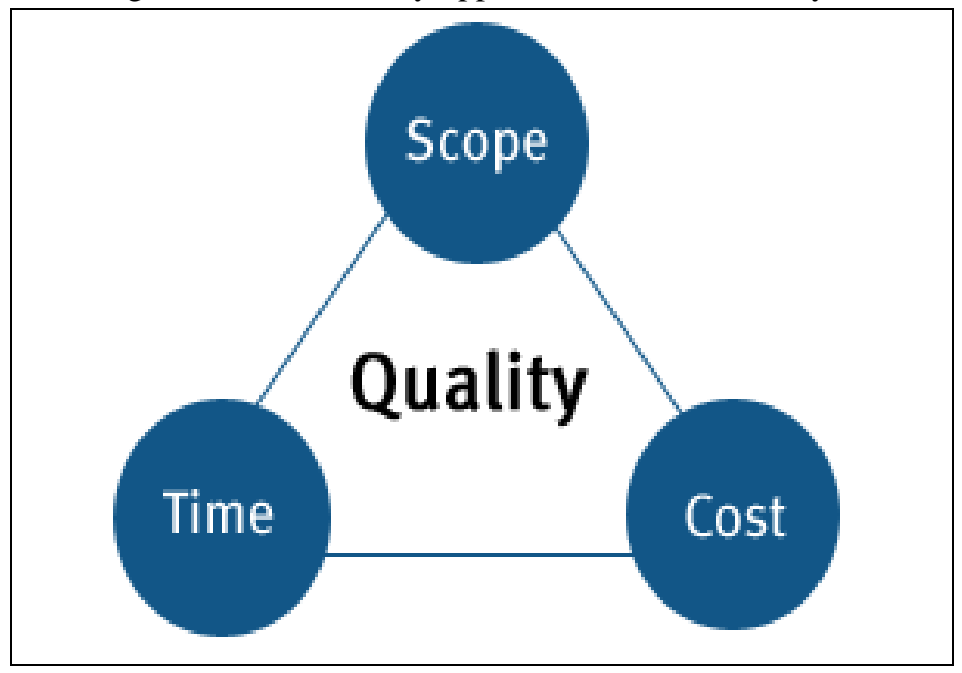

Figure 3: The Project Management Triangle places the three attributes scope, cost, and time on the corners of a triangle to show that it is not possible to optimize all three - one will always suffer.

\section{A. Scope}

For organizational projects with the goal of implementing an IT tool, the scope, or the expected results of a project, is often equated with the use cases or features of the tool. However, since our project was not the DMS, but the introduction of a DMS, we can differentiate between the DM Process Scope and the DM System Scope. The DM System Scope consists of the requirements for the DM system, as they are modeled in DOORS. The DM Process Scope comprises all the activities around the technical implementation of the system, from project management over user surveys to user trainings. Bear in mind that the goal of our project was not only the implementation of a DMS, but a successful introduction of said DMS as well.

Regarding the process scope and in order to optimize the percentage of contented users, we have undertaken the following measures along the process to optimize the success of the DMS introduction project regardless of the resulting system:

1. Project Management: although it seems to be an obvious hint that good projects should include project management, too many projects seem to be conducted without any project management whatsoever in practice. Hence we would like to stress that it is important to manage the project, if it shall be successful, and not only the project, but also the project quality, and the project's budget. Defining the project change management at an early stage also is a good practice.

2. Process organization: with organizational projects regarding the introduction of an IT tool, it is important to define the process at an early stage, in order to avoid the trap of only thinking of how the IT tool will work and how it can be used. The tool should follow the process, not the other way around. Organizing the process also means to 
write down requirements for interfacing processes and tools, in this case, like the CM process. Time should be allocated to update the process descriptions, which can be more time-consuming than one might assume in the beginning, but will be a by-product of a good process organization strategy.

3. Project Marketing: The best of IT tools is useless if the tool's users do not want to use it. Therefore, it is crucial to involve the users (or a selected subset, some key persons in a DM expert users group who will serve as multipliers) from the beginning of the project. Properly prepared requirements workshops with the users are a good start to make sure that all the required features are thought of before the implementation. If users have to work with a system that does not have the features that they deem useful and necessary, they are less likely to work with the system, which can make the system a waste of time and money in the worst case. The more users participate in the requirements workshops, the less likely it is that important features will be forgotten, and the fewer users will be in a position to complain that the system does not satisfy their needs. Project marketing also means to keep the users informed during the project, and ideally to let them try out test installations of the final tools from which the winner must be selected. User training is an important aspect of project marketing as well, with the additional benefit that the probability of the user engaging successfully with the system improves markedly.

4. Requirements Management in detail is part of the system scope, but it shall be stated in the process scope section that requirements management is an absolute must and that "requirements" means more than only the functional requirements or use cases, but that there are also formal requirements to be addressed.

5. Test Management: allocate time for tests, be they functional tests, test system implementations with the option for future users to test the system, or formal acceptance tests

6. Migrate data: plan for an efficient data migration from previous tools or storage locations, e.g., the CMS or file shares.

The system scope for an IT system matches closely with the system requirements, thus managing system requirements in DOORS or a similar system is highly advisable. We have started the DMS introduction project with two user workshops, where the requirements to be fulfilled by the DMS were collected and discussed. System requirements include first and foremost functional requirements, i.e., use cases, but a number of other requirements and constraints must not be forgotten in order to optimize the project success:

1. Functional Requirements: use cases of the process in question, here DM. The use cases can be found by imagining the process and by collecting ideas from the users as to how the process is lived at the moment without a central IT tool.

2. Interface Requirements: A systematic analysis of interfacing tools and processes, as mentioned above, will yield some interface requirements, others may be deducted from functional requirements or be thought of by users.

3. Performance Requirements: the usability of a system will invariably be judged poorly if the user feels that the system is slow and makes him wait. Performance requirements should be specified to avoid poor system performance regarding speed, reliability, availability, and traceability.

4. Design Constraints: include standards compliance to ECSS and applicable ISO ${ }^{3}$ standards. In European space operations missions, ECSS standards compliance is almost always a must. By designing the DMS in such a way that ECSS standards will be fulfilled automatically, we have both secured the acceptance of the DMS and simplified its applicability and practical use to future projects. The need to fill out ECSS compliance matrices for every new mission is mitigated if the standard DM tool is already known to be ECSS compliant. Security constraints arising from standards can be addressed here or under attributes, see below.

The ECSS standards requirements applicable here include the fact that the cover page of a document must visually indicate who has approved it, and which document status the document has. This was taken into account when designing the approval workflow and the metadata fields.

5. Attributes: the attributes availability, confidentiality, maintainability, and transferability/conversion relate to the performance requirements and qualify the system with regard to potential problems. The role access model and other document access properties are specified under the confidentiality attribute.

6. Other Requirements: any requirements which do not fit under the previous headings shall be listed here, in our case requirements as to how the DMS shall be operated.

7. Addressing all challenges and constraints listed in Section II. and III. is a good way of verifying that all system requirements have been thought of. The constraints which apply to the tool instead of the process shall be reflected among the requirements. 


\section{B. Time}

In hindsight, we have chosen to optimize scope and cost over time, although one could argue that this preference came about from a natural boundary, which was the company which implemented the DMS customizing and took much longer than announced. Since the company had offered the customization at a fixed price, this did not affect the cost criterion too greatly, but the time criterion did suffer. However, in combination with the effect discussed above, namely that users like the idea of migrating their documentation to a new, clean, and useful DMS, but dislike the idea of sorting years' worth of old and redundant documentation, we can conclude that the lost time did not cause any problems.

\section{Cost}

Since we have selected an open source DMS instead of a proprietary one, the costs were still better than they could have been for a proprietary system which would have satisfied all our requirements (scope). The cost for the necessary customizations was higher than it would have been for one of the proprietary systems, which has a lot of the requested (and many unrequested) features built in already, but therefore has a much higher off-the-shelf cost. Some of the proprietary systems which had been offered to us had to be declined because they exceeded our budget.

We also employed the cost factor to gain information about the bidding companies. Where suspiciously cheap bids and subsequent discussions with the bidders lead us to believe that they had actually not grasped the challenge correctly, such bids were excluded from the final selection process.

\section{Conclusion}

In this paper, we have discussed how we have reaped expected benefits while addressing the expected and arising challenges in the process of successfully implementing a DMS. Special care was given to the system's usability in order to achieve maximum acceptance among the users, while also satisfying the quality and security constraints for a DMS used for space operations. One important further step is the final implementation of the external access to the system, which will further improve the users' opinion of the system. Another further step is to continue the project marketing by requesting feedback and acting upon improvement suggestions or complaints, in short, by continuing good issue tracking and change management. This approach, especially the project management part of it, is applicable to any organizational project introducing an IT system within an organization that engages in space operations with the given security constraints.

\section{Acknowledgments}

I would like to thank Dr. Josef Friedrich for his constant support and helpful knowledge, for being a general joy to work with and for proof-reading this paper. Dr. Thomas Bassler deserves my thanks both for continuous good consultations and for proof-reading this paper. I thank my sister, Dr. Natascha Perera, for helpful comments.

\section{References}

Books

${ }^{1}$ Michael W. Newell, Marina N. Grashina, The Project Management Question and Answer Book, 2004, p.8

Standards

${ }^{2}$ ECSS, ESA-ESTEC, ECSS-M-ST-40 C, Configuration and information management, Third issue Revision 1, 06 March 2009, Noordwijk, The Netherlands.

${ }^{3}$ ISO/IEC, 27001:2013 Information technology - Security techniques - Information security management systems Requirements (second edition). 